\title{
Erratum: Variability of collective dynamics in random tree networks of strongly coupled stochastic excitable elements [Phys. Rev. E 98, 052303 (2018)]
}

\author{
Ali Khaledi-Nasab, Justus A. Kromer, Lutz Schimansky-Geier, and Alexander B. Neiman
}

(Received 5 June 2019; published 21 June 2019)

DOI: 10.1103/PhysRevE.99.069902

Figures 8(a) and 8(b) in this paper are erroneous due to a mistake in the numerical code for the identification of the leaf nodes. We provide the correct Figs. 8(a) and 8(b) below. With the correct code, the clusters of the threshold current observed in the weak-coupling regime for the full binary trees disappear [Fig. 8(a)]. This error does not affect the rest of the results, including the stochastic dynamics, the strong-coupling limit predictions, and the conclusion, which remain intact.
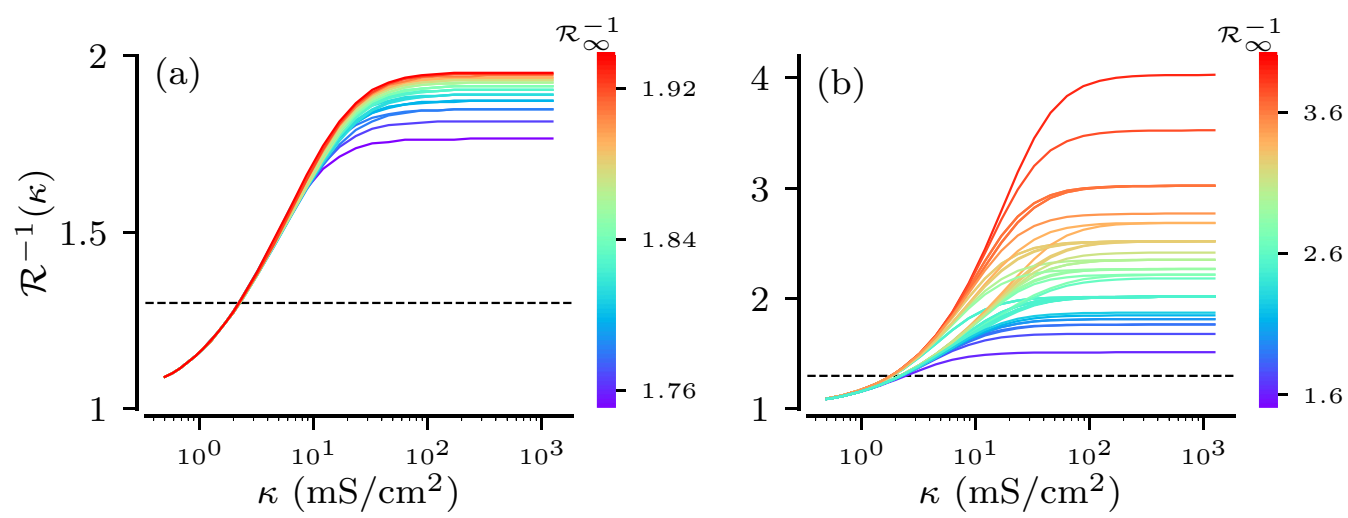

FIG. 8. Normalized threshold current, $\mathcal{R}^{-1}\left(\kappa,\left\{\mathcal{D}_{k}\right\},\left\{h_{k}\right\}\right)=J_{\mathrm{th}}\left(\kappa,\left\{\mathcal{D}_{k}\right\},\left\{h_{k}\right\}\right) / J_{\mathrm{AH}}$ as a function of coupling strength $\kappa$ for two tree network ensembles. (a) Curves for all 25 full binary trees of Fig. 4 (of our paper). (b) The same for 50 general binary trees with all possible numbers of nodes and leaf nodes with a maximal number of generations $G=3$. The curves are color coded according to the tree's normalized threshold currents in the strong-coupling limit $\mathcal{R}_{\infty, k}^{-1}=\mathcal{N}_{k} / \mathcal{H}_{k}$. The dashed horizontal lines show the value of the applied constant current $J=38.5 \mu \mathrm{A} / \mathrm{cm}^{2}$ used for stochastic simulations in Fig. 9 (of the original paper). 